\title{
Article \\ Contrast Analysis of Polarization in Three-Beam Interference Lithography
}

\author{
Fuping Peng ${ }^{1,2} \mathbb{D}$, Jing $\mathrm{Du}^{2}$, Jialin Du ${ }^{1,2}$, Simo Wang ${ }^{1,2}$ and Wei Yan ${ }^{2, *(\mathbb{C}}$ \\ 1 University of Chinese Academy of Sciences, Beijing 100049, China; pengfuping16@mails.ucas.ac.cn (F.P.); \\ dujialin18@mails.ucas.ac.cn (J.D.); wangsimo18@mails.ucas.ac.cn (S.W.) \\ 2 Institute of Optics and Electronics, Chinese Academy of Sciences, Chengdu 610209, China; dujing@ioe.ac.cn \\ * Correspondence: yanwei@ioe.ac.cn
}

check for updates

Citation: Peng, F.; Du, J.; Du, J.; Wang, S.; Yan, W. Contrast Analysis of Polarization in Three-Beam Interference Lithography. Appl. Sci. 2021, 11, 4789. https://doi.org/ 10.3390/app11114789

Received: 29 April 2021

Accepted: 18 May 2021

Published: 23 May 2021

Publisher's Note: MDPI stays neutral with regard to jurisdictional claims in published maps and institutional affiliations.

Copyright: (c) 2021 by the authors. Licensee MDPI, Basel, Switzerland. This article is an open access article distributed under the terms and conditions of the Creative Commons Attribution (CC BY) license (https:// creativecommons.org/licenses/by/ $4.0 /)$.

\begin{abstract}
This paper analyzes the effect of polarization and the incident angle on the contrasts of interference patterns in three-beam interference lithography. A non-coplanar laser interference system was set up to simulate the relationship between contrast, beam polarization, and the incident angle. Different pattern periods require different incident angles, which means different contrast losses in interference lithography. Two different polarization modes were presented to study the effects of polarization with different incident angles based on theoretical analysis simulations. In the case of the co-directional component TE polarization mode, it was demonstrated that the pattern contrast decreases with the increase in the incident angle and the contrast loss caused by the polarization angle error also grew rapidly. By changing the mode to azimuthal (TE-TE-TE) polarization, the contrast of the interference pattern can be ensured to remain above 0.97 even though the incident angle is large. In addition, TE-TE-TE mode can accept larger polarization angle errors. This conclusion provides a theoretical basis for the generation of high-contrast light fields at different incident angles, and the conclusion is also applicable to multi-beam interference lithography.
\end{abstract}

Keywords: pattern contrast; polarization; incident angle; interference lithography

\section{Introduction}

As a new type of material with a photonic band gap, photonic crystals play an important role in modern optical systems, such as photonic crystal fibers, waveguides, lowthreshold lasers, multifunctional sensors, cavity quantum electrodynamics, and quantum information processing [1-6]. Over the past decades, many techniques have been developed for the fabrication of photonic crystals, of which the laser interference lithography (LIL) is a powerful technology due to its low cost, short time consumption, and lack of necessity for precise focusing. LIL was first used to fabricate one-dimensional (1D) grating using double-beam interference. With the addition of a third or further beams, 2D and 3D lattice interference patterns can be generated, including all 2D and 3D Bravais lattice structures [7-11].

Since the energy of the interference fringes is sinusoidally distributed, a high-contrast LIL can write sub-diffraction-limited features in photoresist [12,13]. This is possible because of the non-linear response of positive photoresist to the light dose. The feature size (line width) is reduced by overexposing the high-contrast sinusoidal energy distribution light field $[14,15]$. Therefore, the actual limit of the line width is set by the light field contrast and the sensitivity of the photoresist. In order to obtain the smallest possible feature size, the contrast of the interference light field needs to be sufficiently high. To ensure sufficient interference contrast, the design of the interferometer and the selection of the beam parameters are crucial.

As shown in the following papers [16-18], the source of the contrast loss of the double-beam interference in the same plane mainly includes the beam pointing deviation, the polarization state, and the intensity ratio of the interference beam. Different from 
double-beam interference, multi-beam interference lithography (MIL) is the superposition of the intensity of multiple groups of non-coplanar interference with different periods, directions, and extremum [19-21]. The value of the incident angle controls the period of the interference pattern. For non-coplanar interference, the contrast of the interference light field is simultaneously affected by the azimuth angle, incident angle, and polarization state of each beam [22-24]. Therefore, it is necessary to select a suitable combination of beam polarization states for different incident angles to obtain the best contrast and consider the influence of the beam pointing stability on the contrast under that incident combination.

In this paper, we discuss the two-beam non-coplanar interference, study the relationship between fringe visibility and the spatial incidence direction of the two beams, and analyze the source of the contrast loss in the non-coplanar interference. We calculate how to select the appropriate combination of beam polarization states to ensure sufficient contrast under different incident angles. Then, according to the non-coplanar interference model, we demonstrate the specific beam polarization combinations that should be used for different pattern periods in three-beam LIL. Although the focus of this paper is three-beam LIL, the same analysis of contrast loss and the beam polarization combination mode is applicable for N-beam $(n>3)$ interference lithography.

\section{Contrast in Double-Beam Non-Coplanar Interference Patterns}

When two laser beams from the same light source coincide, an interference pattern is produced, which contains all of the information including the intensity and phase of the two interfering beams. The interference of two beams is the superposition of electric field vectors. The electric field vector of $i$ th beam vector can be written as

$$
\boldsymbol{E}_{\boldsymbol{i}}=A_{i} \boldsymbol{e}_{i} \exp \left[\mathbf{i}\left(\boldsymbol{k}_{i} \cdot \boldsymbol{r}+\varphi_{i}\right)\right]
$$

where $A_{i}$ is the amplitude, $\boldsymbol{e}_{i}$ is the polarization vector, $\boldsymbol{k}_{i}$ is the wave propagation vector, $r$ is the position vector at interference area, and $\varphi_{i}$ is the initial phase of the light wave. The intensity of two-beam interference can be calculated as follows:

$$
I_{2-\text { beam }}=\left(\boldsymbol{E}_{1}+\boldsymbol{E}_{2}\right)\left(\boldsymbol{E}_{1}+\boldsymbol{E}_{2}\right)^{*}=\left|A_{1}\right|^{2}+\left|A_{2}\right|^{2}+2 A_{1} A_{2}\left|\boldsymbol{e}_{1} \boldsymbol{e}_{2}^{T}\right| \cos \left[\left(\boldsymbol{k}_{1}-\boldsymbol{k}_{2}\right) \cdot \boldsymbol{r}+\left(\varphi_{1}-\varphi_{2}\right)\right]
$$

Interference contrast is defined as

$$
K=\frac{I_{\max }-I_{\min }}{I_{\max }+I_{\min }}
$$

$I_{\max }$ and $I_{\min }$ represent the minimum and the maximum intensity of the interference field. Substituting Equation (2) into Equation (3) yields

$$
K \propto \frac{2 A_{1} A_{2}\left|e_{1} e_{2}^{T}\right|}{\left|A_{1}\right|^{2}+\left|A_{2}\right|^{2}}
$$

Equation (4) states that contrast is affected by the intensity mismatch and the polarization states for each beam. We already know that the contrast loss caused by the unequal amplitude of the two beams is very low. Even if the amplitude ratio of the two beams reaches 2:3, the contrast loss is only 0.076 . Therefore, the main source of contrast loss for non-coplanar interference is the polarization state of the two beams.

In Equation (1), according to the coordinate rotation transformation matrix $\boldsymbol{e}_{i}, \boldsymbol{k}_{i}$ and $\boldsymbol{r}$ can be expressed as

$$
\begin{gathered}
\boldsymbol{e}_{i}=\left[\begin{array}{l}
\boldsymbol{e}_{i x} \\
\boldsymbol{e}_{i y} \\
\boldsymbol{e}_{i z}
\end{array}\right]^{T}=\left[\begin{array}{c}
\boldsymbol{i}\left(\cos \alpha_{i} \cos \theta_{i} \cos \phi_{i}-\sin \alpha_{i} \sin \phi_{i}\right) \\
\boldsymbol{j}\left(\sin \alpha_{i} \cos \theta_{i} \cos \phi_{i}+\cos \alpha_{i} \sin \phi_{i}\right) \\
-\boldsymbol{k} \sin \theta_{i} \cos \phi_{i}
\end{array}\right]^{T} \\
\boldsymbol{k}_{i}=\left[\begin{array}{lll}
\boldsymbol{k}_{i x} & \boldsymbol{k}_{i y} & \boldsymbol{k}_{i z}
\end{array}\right]=k\left[\begin{array}{lll}
\boldsymbol{i} \cos \alpha_{i} \sin \theta_{i} & \boldsymbol{j} \sin \alpha_{i} \sin \theta_{i} & -\boldsymbol{k} \cos \theta_{i}
\end{array}\right]
\end{gathered}
$$




$$
\boldsymbol{r}=\left[\begin{array}{lll}
\boldsymbol{i x} & j y & \boldsymbol{k} z
\end{array}\right]^{T}
$$

Here, $\alpha_{i}$ and $\theta_{i}$ are the azimuthal angle and the incident angle, respectively; $\phi_{i}$ is the polarization angle; $k=2 \pi / \lambda$ is the wavenumber; and $\lambda$ is the wavelength of the laser. As shown in Figure 1, the azimuth angles of the two non-coplanar coherent beams are $\alpha_{1}=0$ and $\alpha_{2}=\alpha(0<\alpha<\pi)$, and they incident at the identical angle $\theta$. When $\alpha$ is equal to $\pi$, it turns into coplanar interference. Using Equations (5)-(7), the contrast for two-beam interference can be further reduced to

$$
K \propto\left|\begin{array}{c}
\sin \alpha \cos \theta \sin \left(\phi_{1}-\phi_{2}\right)+\cos \alpha \cos \left(\phi_{1}-\phi_{2}\right) \\
+(1-\cos \alpha) \sin ^{2} \theta \cos \phi_{1} \cos \phi_{2}
\end{array}\right|
$$

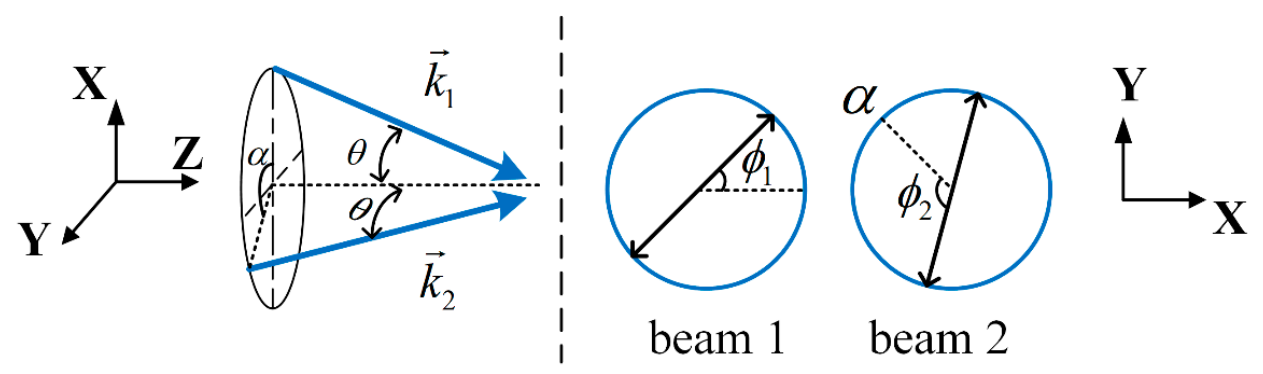

Figure 1. Wave vectors and the coordinate system for double beam interference. Both incident directions of the two beams are on the cone line of the conical surface.

Equation (8) shows that for different azimuth and incident angles, we need to choose different beam polarization modes to maximize the contrast of the interference field.

Owing to the incident surfaces of the two light waves not being coplanar, there is no case where the horizontal polarization component is totally in the same direction. We can only reduce the contrast loss by ensuring that their co-directional TE vibration components achieve the maximum value. Under this condition, the azimuth and the polarization angles of the two beams satisfy the following equation:

$$
\phi_{2}=-\alpha+\phi_{1}
$$

In this case, the contrast can simplify to

$$
K \propto\left|\cos ^{2} \alpha+\sin ^{2} \theta(1-\cos \alpha) \cos \phi_{1} \cos \left(\alpha-\phi_{1}\right)-\cos \theta \sin ^{2} \alpha\right|
$$

From Equation (10), each azimuth angle has a unique polarization co-direction angle corresponding to it, which causes the contrast to obtain a maximum value. We can calculate that the condition for maximum contrast is

$$
\phi_{1}=\frac{\alpha}{2}
$$

This condition is verified in Figure 2. We simulated the contrast of all linear polarization orientations when the azimuth angles were $30^{\circ}, 60^{\circ}, 90^{\circ}, 120^{\circ}, 150^{\circ}$ and $180^{\circ}$, and found that the corresponding polarization angle when the maximum contrast is achieved was $15^{\circ}, 30^{\circ}, 45^{\circ}, 60^{\circ}, 75^{\circ}$, and $90^{\circ}$. This shows that when the polarization angle is on the intersection of the vibration planes of two waves, the contrast is maximized. By comparing Figure $2 a, b$, it can be seen that the polarization orientation has a great influence on the contrast, especially under the condition of a large angle of incidence. 


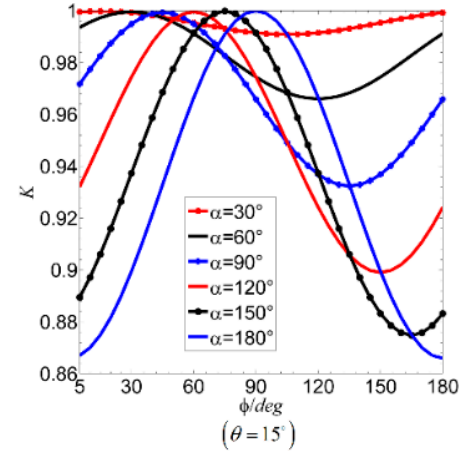

(a)

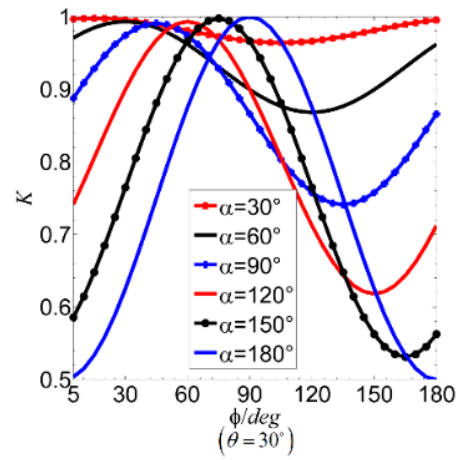

(b)

Figure 2. The relationship between the contrast and the polarization angle combinations with 6 azimuth angles. The interference contrast is most affected by polarization when the azimuth angles are orthogonal. The greater the incident angle, the greater the contrast loss caused by the polarization. For (a), the incident angle is $15^{\circ}$, and for (b), the incident angle is $30^{\circ}$.

Substituting Equation (11) into Equation (10), the maximum contrast that can be achieved by double-beam interference is

$$
K_{\max } \propto 1-\left(\frac{1}{2} \sin ^{2} \theta+\cos ^{2} \theta+\cos \theta\right) \sin ^{2} \alpha
$$

As shown in Figure 3, only in the case of coplanar interference $(\alpha=\pi)$ and where two beams are incident in TE-TE mode will the contrast not decrease due to the increase in the incident angle. As the azimuth angle increases, the contrast loss caused by large incident angle interference is considerable until it increases to $90^{\circ}$, where the contrast loss caused by large incident angle interference reaches the maximum. When the azimuth angle continues to increase beyond $90^{\circ}$, the contrast loss gradually decreases. Until coplanar interference is reached, the contrast loss caused by the large incident angle completely disappears. As long as the incident angle is not greater than $35^{\circ}$, the contrast loss of any azimuth non-coplanar interference does not exceed 0.02. However, if the incident angle continues to increase, the contrast sharply decreases.

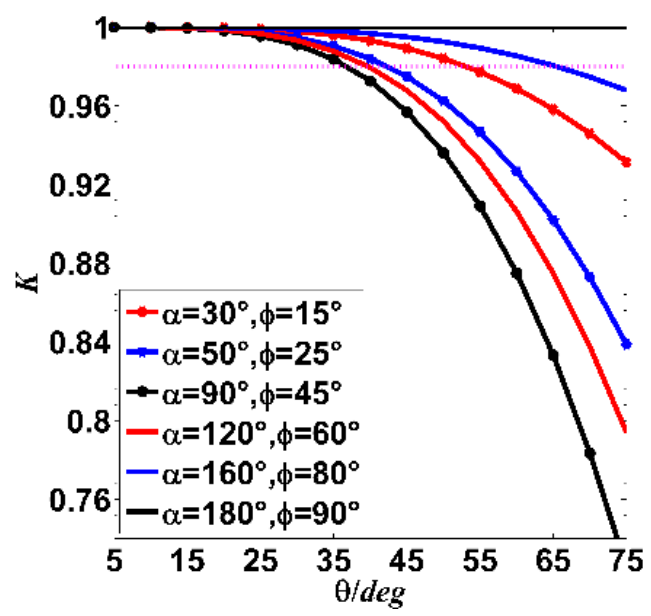

Figure 3. The relationship between the contrast of the two-beam interference light field and the incident angle under the optimal polarization incident condition. In the case of non-coplanar interference of two beams, the contrast loss caused by the increase in the incident angle is inevitable. 
In addition, the existence of polarization angle deviation will inevitably cause the loss of contrast. To illustrate this impact, we assume that the polarization angle deviations of the two beams are $\delta_{1}$ and $\delta_{2}$. In this case, the contrast can be described as

$$
K \propto\left|\begin{array}{c}
\sin \alpha \cos \theta \sin \left(\delta_{1}-\delta_{2}\right)+\cos \alpha \cos \left(\delta_{1}-\delta_{2}\right) \\
+\frac{1}{2}(1-\cos \alpha) \sin ^{2} \theta \cos \left(\delta_{1}-\delta_{2}\right) \cos \left(\alpha+\delta_{1}-\delta_{2}\right)
\end{array}\right|
$$

Under the same incident angle, the contrast loss is largest when the azimuth angle is equal to $90^{\circ}$. The polarization angle deviation at this point further increases the contrast loss. As shown in Figure $4 \mathrm{a}, \mathrm{b}$, when the polarization angle deviation of the two beams reaches $5^{\circ}$, the greater the incident angle, the greater the loss of contrast.

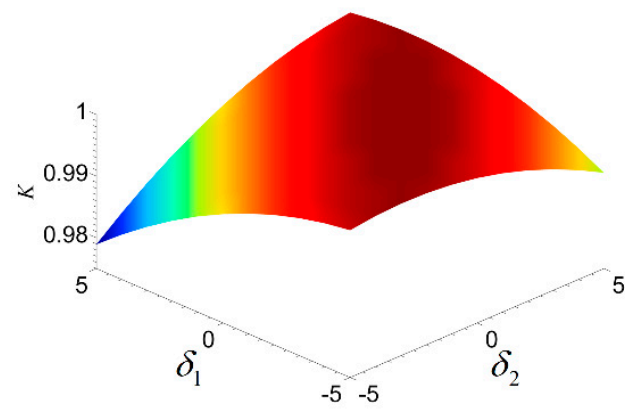

(a) $\theta=15^{\circ}$

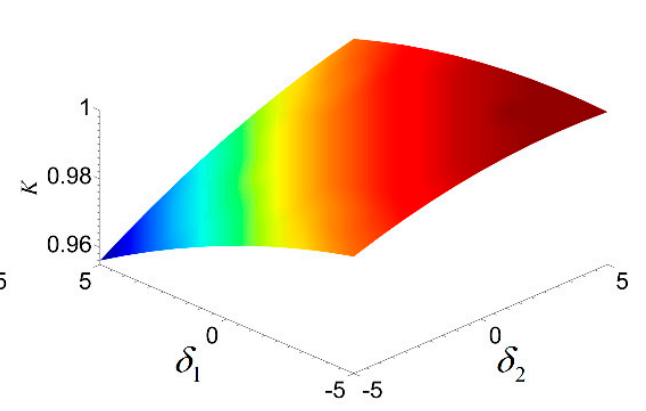

(b) $\theta=30^{\circ}$

Figure 4. Contrast loss caused by polarization angle deviation at different incident angles. The larger the incident angle, the more unstable the interference system. For (a), the incident angle is $15^{\circ}$. For (b), the incident angle is $30^{\circ}$.

The incident angle, azimuth, and polarization for non-coplanar interference contrast were analyzed in turn. As shown in Figure 5, considering all factors at a time, we obtained the contrast of two-beam non-coplanar interference at different incident angles, azimuth angles, and polarization angle deviations. The Figure shows that the polarization orientation must be adjusted to reduce the contrast loss caused by the increase in the incident angle. The maximum co-directional polarization component mode is not suitable for large-angle incident situations.

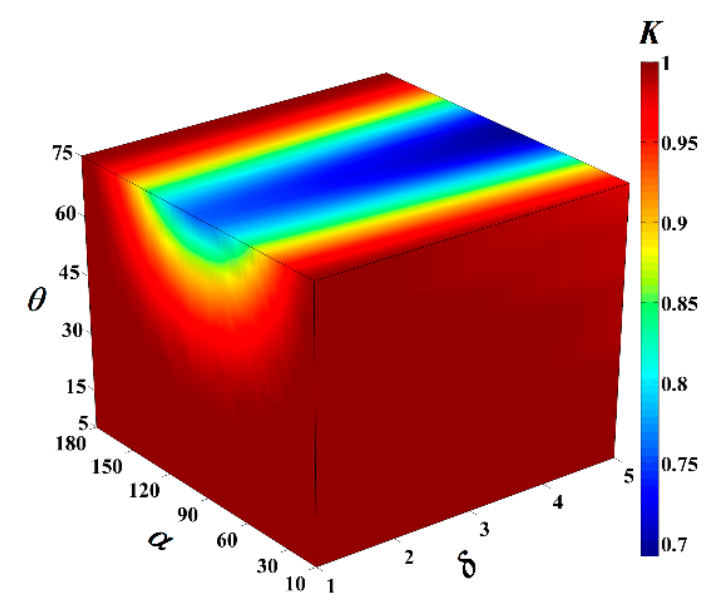

Figure 5. The contrast of two-beam non-coplanar interference at different incident angles, azimuth angles, and polarization angle deviations. The polarization mode of the incident light beam is the maximum co-directional polarization component.

\section{Contrast in Three-Beam Interference Patterns}

$N$-beam interference is the incoherent superposition of $N(N-1) / 2$ groups of twobeam non-coplanar interference. Therefore, the above analysis of non-coplanar interference 
is applicable to $\mathrm{N}$-beam interference. The intensity of $\mathrm{N}$-beam interference can be calculated as

$$
I_{N-\text { beam }}=\sum_{i=1}^{N} A_{i}^{2}+2 \sum_{i<j}^{N} A_{i} A_{j} \boldsymbol{e}_{i} \boldsymbol{e}_{j}^{T} \cos \left(\left(\boldsymbol{k}_{i}-\boldsymbol{k}_{j}\right) \cdot \boldsymbol{r}+\left(\varphi_{i}-\varphi_{j}\right)\right)
$$

Regardless of the influence of amplitude and assuming that the initial phases are zero in Equation (14), substituting Equation (14) into Equation (3) yields

$$
K \propto \sum_{i<j}^{N} e_{i} e_{j}^{T}
$$

It can be seen from Equation (15) that the polarization combination of the beams has a key effect on the contrast of the light field. Three-beam interference is a typical case of $\mathrm{N}$-beam interference, the related derivation process, and results are generally applicable in multi-beam interference. Therefore, this paper chooses three-beam interference as the contrast loss analysis model.

\subsection{Co-Directional Component Polarization}

When the polarization common component is the largest, the relationship between the azimuth and the polarization angle of $\mathrm{N}$-beam interference is as follows:

$$
\alpha_{i}=2 \pi \frac{i-1}{N}, \phi_{i}=\phi-\alpha_{i}
$$

where $\phi$ is the polarization angle of the beam whose azimuth is zero. The polarization vector can be written as follows:

$$
\boldsymbol{e}_{i}=\left[\begin{array}{c}
i\left(\cos \theta \cos \left(2 \pi \frac{i-1}{N}\right) \cos \left(\phi-2 \pi \frac{i-1}{N}\right)-\sin \left(2 \pi \frac{i-1}{N}\right) \sin \left(\phi-2 \pi \frac{i-1}{N}\right)\right) \\
j\left(\cos \theta \sin \left(2 \pi \frac{i-1}{N}\right) \cos \left(\phi-2 \pi \frac{i-1}{N}\right)+\cos \left(2 \pi \frac{i-1}{N}\right) \sin \left(\phi-2 \pi \frac{i-1}{N}\right)\right. \\
-k \sin \theta \cos \left(\phi-2 \pi \frac{i-1}{N}\right)
\end{array}\right]^{T}
$$

As shown in Figure 6, the three incident beams follow a symmetrical configuration with the azimuth of $\alpha_{1}=0^{\circ}, \alpha_{2}=120^{\circ}$, and $\alpha_{3}=240^{\circ}$. The incident angles of the three beams are set as $\theta_{1}=\theta_{2}=\theta_{3}=\theta$. The polarization vectors of the three beams are

$$
\begin{aligned}
& \boldsymbol{e}_{1}=\left[\begin{array}{lll}
i \cos \theta \cos \phi & j \sin \phi & -k \sin \theta \cos \phi
\end{array}\right] \\
& \begin{array}{c}
\boldsymbol{e}_{2}=\left[\begin{array}{c}
i\left(\frac{1}{4} \cos \theta \cos \phi-\frac{\sqrt{3}}{4} \cos \theta \sin \phi+\frac{\sqrt{3}}{4} \sin \phi+\frac{3}{4} \cos \phi\right) \\
j\left(-\frac{\sqrt{3}}{4} \cos \theta \cos \phi+\frac{3}{4} \cos \theta \sin \phi+\frac{1}{4} \sin \phi+\frac{\sqrt{3}}{4} \cos \phi\right) \\
k\left(\frac{1}{2} \sin \theta \cos \phi-\frac{\sqrt{3}}{2} \sin \theta \sin \phi\right)
\end{array}\right]^{T} \\
\boldsymbol{e}_{3}=\left[\begin{array}{c}
i\left(\frac{1}{4} \cos \theta \cos \phi+\frac{\sqrt{3}}{4} \cos \theta \sin \phi-\frac{\sqrt{3}}{4} \sin \phi+\frac{3}{4} \cos \phi\right) \\
j\left(\frac{\sqrt{3}}{4} \cos \theta \cos \phi+\frac{3}{4} \cos \theta \sin \phi+\frac{1}{4} \sin \phi-\frac{\sqrt{3}}{4} \cos \phi\right) \\
k\left(\frac{1}{2} \sin \theta \cos \phi+\frac{\sqrt{3}}{2} \sin \theta \sin \phi\right)
\end{array}\right]^{T}
\end{array}
\end{aligned}
$$

The three beams are symmetrically incident, and the vibration components of the $x-y$ plane are along the same angle $\phi$. Substituting Equation (18) into Equation (15), considering the polarization and incident angle one at a time, the contrast as a function of two factors is plot in Figure 6. Similar to the two-beam non-coplanar interference, when interfering at a small angle, the polarization angle will not change the contrast. The contrast loss will only increase as the incident angle increases. As the incident angle continues to increase, the contrast is more sensitive to the changes in the polarization angle and is extremely susceptible to polarization angle deviations. 

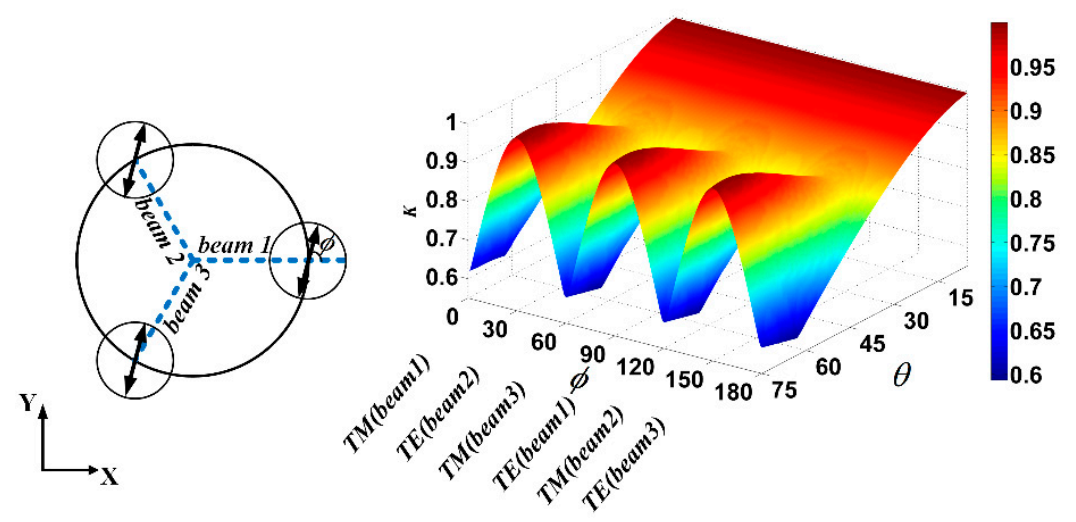

Figure 6. On the left is a top view of three beams incident, and the arrows represent the polarization direction of the beams on the $x-y$ plane. The figure on the right shows that the maximum co-directional polarization component is still applicable to the small-angle incident situation of multi-beam interference. However, as the incident angle increases, the stability of the multi-beam interference system will be very poor.

In this case, taking the TE wave of one of the three beams as the common vibration direction can ensure that the contrast is sufficiently high even at a large incident angle. The polarization angle of beam 1 is $\phi_{1}=\phi=90^{\circ}$. From Equation (16), the polarization vectors can be expressed as

$$
\begin{gathered}
\boldsymbol{e}_{1}=\left[\begin{array}{lll}
0 & j & 0
\end{array}\right] \\
\boldsymbol{e}_{2}=\left[\begin{array}{lll}
i\left(-\frac{\sqrt{3}}{4} \cos \theta+\frac{\sqrt{3}}{4}\right) & j\left(\frac{3}{4} \cos \theta+\frac{1}{4}\right) & k\left(-\frac{\sqrt{3}}{2} \sin \theta\right)
\end{array}\right] \\
\boldsymbol{e}_{3}=\left[\begin{array}{lll}
i\left(\frac{\sqrt{3}}{4} \cos \theta-\frac{\sqrt{3}}{4}\right) & j\left(\frac{3}{4} \cos \theta+\frac{1}{4}\right) & k\left(\frac{\sqrt{3}}{2} \sin \theta\right)
\end{array}\right]
\end{gathered}
$$

From Equation (14), the intensity distribution of three-beam interference can be calculated by

$$
\begin{aligned}
I(x, y, z)= & 3+(3 \cos \theta+1) \cos \left(\frac{3}{2} k \sin \theta \cdot x\right) \cos \left(\frac{\sqrt{3}}{2} k \sin \theta \cdot y\right) \\
& +\left[\frac{9}{4}\left(\cos \theta+\frac{1}{3}\right)^{2}-2\right] \cos (\sqrt{3} k \sin \theta \cdot y)
\end{aligned}
$$

According to the above equation, the maximum intensity of the interference field is related to the value of the incident angle. As shown in Figure 7, after the incident angle is increased to $32^{\circ}$, the contrast no longer decreases as the incident angle increases. At the same time, with the increase in incident angle, the pattern transitions from the hexagonal center maximum lattice to the hexagonal minimum lattice. The maximum value of the interference field intensity gradually decreases to half of the theoretical maximum value. This change in the maximum intensity value undoubtedly increases the difficulty of determining the exposure time in the photolithography process.

\subsection{TE-TE-TE Polarization}

Both co-directional component TE polarization and co-directional component TM polarization were analyzed above. In both cases, neither the high contrast of the light field nor the stability of the interference system can be guaranteed under the conditions of large-angle interference. As shown in Figure 8d, another polarization mode is proposed for interference lithography under large-angle incidence. 
(a)

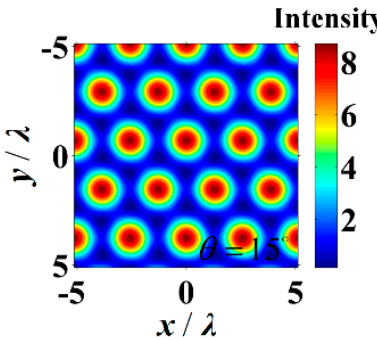

(c)

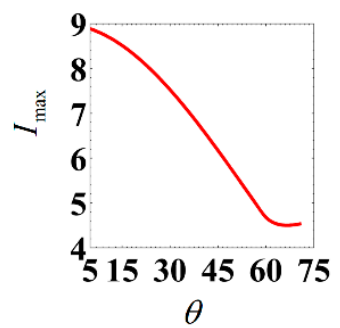

(b)

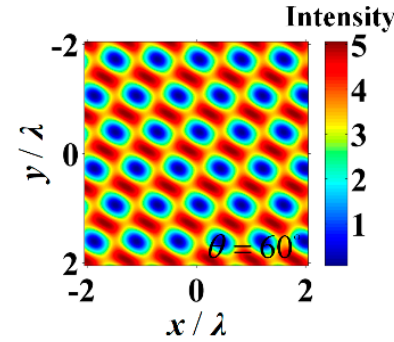

(d)

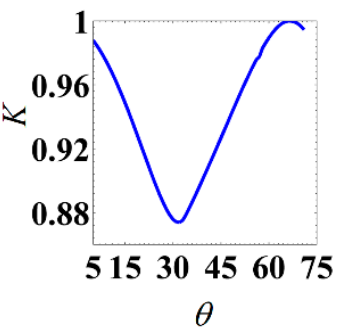

Figure 7. The interference of the three beams incident with the maximum co-directional TE polarization. The light field intensity distribution at different incident angles: the maximum value of the light field intensity changes with the incident angle and the relationship between the contrast and the incident angle. (a) The intensity distribution of the light field with an incident angle equal to $15^{\circ}$. (b) The intensity distribution of the light field with an incident angle equal to $60^{\circ}$. (c,d) The maximum value of the light field intensity and the contrast change due to the incident angle.

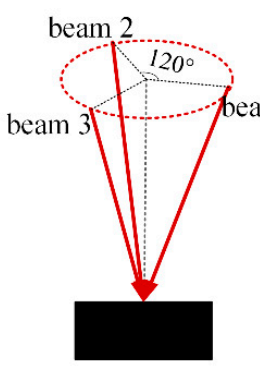

(a)

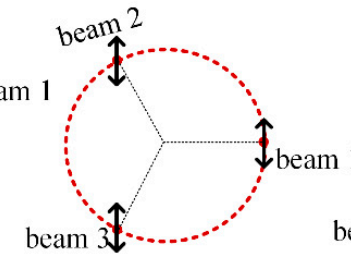

Co-directional component TE polarization

(b)

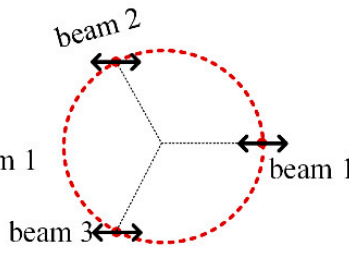

Co-directional component TM polarization

(c)

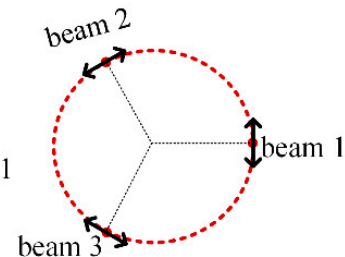

TE-TE-TE

(d)

Figure 8. Schematic diagram of polarization mode of three-beam interference. (a) The incident azimuth of the interference of the three beams. (b-d) Three different types of polarization mode combinations, the arrows represent the polarization direction of the beams on the $x-y$ plane.

When the polarization of the three beams are in TE-TE-TE mode, the polarization angle of three beams is $\phi_{1}=\phi_{2}=\phi_{3}=90^{\circ}$. The polarization vectors can be expressed as

$$
\begin{gathered}
\boldsymbol{e}_{1}=\left[\begin{array}{lll}
0 & j & 0
\end{array}\right] \\
\boldsymbol{e}_{2}=\left[\begin{array}{lll}
-i \frac{\sqrt{3}}{2} & -j \frac{1}{2} & 0
\end{array}\right] \\
\boldsymbol{e}_{3}=\left[\begin{array}{lll}
i \frac{\sqrt{3}}{2} & -j \frac{1}{2} & 0
\end{array}\right]
\end{gathered}
$$

From Equation (14), the intensity distribution of three-beam interference can be calculated by

$$
I(x, y, z)=3-2 \cos \left(\frac{3}{2} k \sin \theta \cdot x\right) \cos \left(\frac{\sqrt{3}}{2} k \sin \theta \cdot y\right)-\cos (\sqrt{3} k \sin \theta \cdot y)
$$

It can be observed from Equation (22) that the maximum intensity remains unchanged at 4.5 when the incident is in TE-TE-TE mode, which is only half of the theoretical value. The increase in the incident angle will not cause additional contrast loss and it makes it 
easier for us to control the exposure dose at different incident angles. Although the increase in exposure time may introduce additional environmental errors to the interference light field, it is clearly easier to obtain a pattern with a smaller feature size when the light field has a high contrast.

It can be seen from Figure 6 that the contrast loss caused by the polarization angle deviation must be considered, especially under the condition of large-angle interference. Under TE-TE-TE incident condition, the polarization angle deviations are $\delta_{1}, \delta_{2}, \delta_{3}$, and the polarization angle of three beams are $\phi_{1}=90^{\circ}+\delta_{1}, \phi_{2}=90^{\circ}+\delta_{2}, \phi_{3}=90^{\circ}+\delta_{3}$. The polarization vector can be expressed as

$$
\begin{gathered}
\boldsymbol{e}_{1}=\left[\begin{array}{lll}
-i \cos \theta \sin \delta_{1} & j \cos \delta_{1} & k \sin \theta \sin \delta_{1}
\end{array}\right] \\
\boldsymbol{e}_{2}=\left[\begin{array}{ccc}
i\left(\frac{1}{2} \cos \theta \sin \delta_{2}-\frac{\sqrt{3}}{2} \cos \delta_{2}\right) & j\left(-\frac{\sqrt{3}}{2} \cos \theta \sin \delta_{2}-\frac{1}{2} \cos \delta_{2}\right) & k \sin \theta \sin \delta_{2}
\end{array}\right] \\
\boldsymbol{e}_{3}=\left[\begin{array}{lll}
i\left(\frac{1}{2} \cos \theta \sin \delta_{3}+\frac{\sqrt{3}}{2} \cos \delta_{3}\right) & j\left(\frac{\sqrt{3}}{2} \cos \theta \sin \delta_{3}-\frac{1}{2} \cos \delta_{3}\right) & k \sin \theta \sin \delta_{3}
\end{array}\right]
\end{gathered}
$$

It can be concluded from Equation (23) that when there is a deviation in the polarization angle, the contrast loss is related to the incident angle. Under the condition that the polarization angle deviations $\delta_{1}, \delta_{2}$ and $\delta_{3}$ are not more than $\pm 5^{\circ}$, we calculated the minimum value of contrast when the incident angle is increased from $5^{\circ}$ to $75^{\circ}$. As shown in Figure 9, when the incident angle is not more than $40^{\circ}$, the contrast loss does not exceed 0.015 . Even if the interference angle reaches $60^{\circ}$, the contrast still has 0.975 . At this time, we have a sufficient contrast loss margin to consider the standing wave effect caused by large angle incidence.

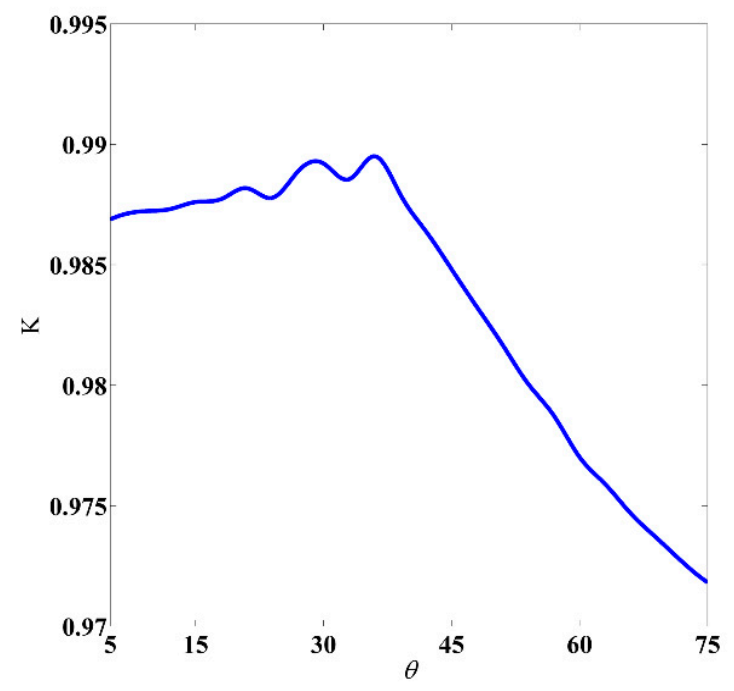

Figure 9. The TE-TE-TE polarization mode is used for three-beam interference, and the contrast at different incident angles is obtained when the polarization direction of the three beams has a deviation of $\pm 5^{\circ}$.

\section{Discussion}

There is a contradiction between narrower line width and higher contrast in LIL. The smaller feature size of the pattern means a larger spatial incident angle, which also brings about the enhancement of the standing wave effect and the sharp decrease in the contrast of the light field. When the coherence of the light source is not considered, the contrast of MIL depends on the polarization state of the beam, the angle of incidence, the deviation of the polarization angle, and the stability of the beam pointing at the same time. In this paper, by constructing a MIL model, we obtained the optimal solution for the high-contrast light field of three-beam interference lithography under different pattern line widths. In the case of a small incident angle interference, the co-directional TE polarization incident combination is adopted, which can reduce the exposure time while obtaining a high-contrast light field. When the incident is at a large angle, the azimuthal TE polarization incident combination 
is used to obtain a high-contrast light field while allowing higher beam pointing stability deviations. This conclusion is also applicable to MIL, which provides a theoretical reference for the generation of narrow linewidth patterns and equipment development in interference lithography.

\section{Conclusions}

In this work, the contrast loss model under non-coplanar interference conditions is proposed. It is demonstrated that the contrast under non-coplanar interference conditions will decrease with the increase in the incident angle. By changing the polarization combination mode of the beams, we avoided a large-scale contrast loss when fabricating narrow linewidth structures by LIL. According to the simulation result data, the deviation of the polarization angle and the polarization mode are important factors that cause a loss of contrast. The TE-TE-TE polarization mode is the best polarization combination for three-beam interference lithography to prepare sub-wavelength structures.

Author Contributions: Conceptualization, F.P., J.D. (Jing Du)., and W.Y.; methodology, F.P. and W.Y.; software, F.P. and S.W.; validation, F.P. and S.W.; formal analysis, F.P. and J.D. (Jialin Du).; writing-original draft preparation, F.P., J.D. (Jing Du). and S.W.; writing-review and editing, F.P. and W.Y.; project administration, W.Y. and J.D. (Jialin Du).; funding acquisition, W.Y. All authors have read and agreed to the published version of the manuscript.

Funding: This research was supported by the Instrument Development of Chinese Academy of Sciences (Nos. YJKYYQ20180008, YJKYYQ20180006), Sichuan Science and Technology Program (Nos. 2020JDJQ0007, 2021JDRC0089, 2021JDRC0084).

Institutional Review Board Statement: Not applicable.

Informed Consent Statement: Not applicable.

Data Availability Statement: Some or all data, models, or code used during the study can be requests directly from the author.

Conflicts of Interest: The authors declare no conflict of interest.

\section{References}

1. Wang, L.; Wang, Z.-H.; Yu, Y.-H.; Sun, H.-B. Laser interference fabrication of large-area functional periodic structure surface. Front. Mech. Eng. 2018, 13, 493-503. [CrossRef]

2. Liu, H.; Luo, Y.; Kong, W.; Liu, K.; Du, W.; Zhao, C.; Gao, P.; Zhao, Z.; Wang, C.; Pu, M. Large area deep subwavelength interference lithography with a $35 \mathrm{~nm}$ half-period based on bulk plasmon polaritons. Opt. Mater. Express $2018,8,199-209$. [CrossRef]

3. Behera, S.; Joseph, J. Design and fabrication of woodpile photonic structures through phase SLM-based interference lithography for omnidirectional optical filters. Opt. Lett. 2017, 42, 2607-2610. [CrossRef]

4. Xiao, Y.; Zhang, Y.; Shi, Y.; Jiang, S. The study on optical lattice formed by four-beam interference. Optik 2016, 127, 10421-10427. [CrossRef]

5. Sang, W.-h.; Lin, L.; Wang, L.; Min, J.-h.; Zhu, J.-j.; Wang, M.-r. GaN-based light emitting diodes on nano-hole patterned sapphire substrate prepared by three-beam laser interference lithography. Optoelectron. Lett. 2016, 12, 178-181. [CrossRef]

6. Seo, J.-H.; Park, J.H.; Kim, S.-I.; Park, B.J.; Ma, Z.; Choi, J.; Ju, B.-K. Nanopatterning by laser interference lithography: Applications to optical devices. J. Nanosci. Nanotechnol. 2014, 14, 1521-1532. [CrossRef]

7. Shimizu, Y. Metrology, Laser Interference Lithography for Fabrication of Planar Scale Gratings for Optical Metrology. Nanomanufacturing 2021, 4, 1-25. [CrossRef]

8. Hou, Y.; Wang, Z.; Hu, Y.; Li, D.; Qiu, R. Capture and sorting of multiple cells by polarization-controlled three-beam interference. J. Opt. 2016, 18, 035401. [CrossRef]

9. Behera, S.; Kumar, M.; Joseph, J. Submicrometer photonic structure fabrication by phase spatial-light-modulator-based interference lithography. Opt. Lett. 2016, 41, 1893-1896. [CrossRef]

10. Xu, J.; Wang, Z.; Zhang, Z.; Wang, D.; Weng, Z. Fabrication of moth-eye structures on silicon by direct six-beam laser interference lithography. J. Appl. Phys. 2014, 115, 203101. [CrossRef]

11. Xia, D.; Ku, Z.; Lee, S.; Brueck, S. Nanostructures and functional materials fabricated by interferometric lithography. Adv. Mater. 2011, 23, 147-179. [CrossRef]

12. Voisiat, B.; Zwahr, C.; Lasagni, A.F. Growth of regular micro-pillar arrays on steel by polarization-controlled laser interference patterning. Appl. Surf. Sci. 2019, 471, 1065-1071. [CrossRef] 
13. Divan, R.; Makarova, O.V.; Skoog, S.; Narayan, R.; Sumant, A.V.; Tang, C.-M.; Moldovan, N. High-aspect-ratio nanoporous membranes made by reactive ion etching and e-beam and interference lithography. Microsyst. Technol. 2014, 20, 1797-1802. [CrossRef]

14. He, J.; Fang, X.; Lin, Y.; Zhang, X. Polarization control in flexible interference lithography for nano-patterning of different photonic structures with optimized contrast. Opt. Express 2015, 23, 11518-11525. [CrossRef] [PubMed]

15. Kusaka, K.; Kurosawa, H.; Ohno, S.; Sakaki, Y.; Nakayama, K.; Moritake, Y.; Ishihara, T. Waveguide-mode interference lithography technique for high contrast subwavelength structures in the visible region. Opt. Express 2014, 22, 18748-18756. [CrossRef] [PubMed]

16. Miller, D.B.; Jones, A.; McLeod, R.R. Contrast analysis in two-beam laser interference lithography. Appl. Opt. 2020, 59, 5399-5407. [CrossRef]

17. Zhang, Z.; Dong, L.; Ding, Y.; Li, L.; Weng, Z.; Wang, Z. Micro and nano dual-scale structures fabricated by amplitude modulation in multi-beam laser interference lithography. Opt. Express 2017, 25, 29135-29142. [CrossRef]

18. Stay, J.L.; Gaylord, T.K. Contrast in four-beam-interference lithography. Opt. Lett. 2008, 33, 1434-1436. [CrossRef]

19. Shimomura, A.; Fukuda, T.; Emoto, A. Analysis of interference fringes based on three circularly polarized beams targeted for birefringence distribution measurements. Appl. Opt. 2018, 57, 7318-7324. [CrossRef]

20. Lü, H.; Chu, C.; You, K.; Zhao, Q.; Wang, X. Fabrication of two-dimensional special photonic crystals by symmetry-lost beam interference lithography. Optik 2017, 140, 25-31. [CrossRef]

21. Chen, X.; Ren, Z.; Shimizu, Y.; Chen, Y.-1.; Gao, W. Optimal polarization modulation for orthogonal two-axis Lloyd's mirror interference lithography. Opt. Express 2017, 25, 22237-22252. [CrossRef] [PubMed]

22. Zhou, H.; Zeng, L. Method to fabricate orthogonal crossed gratings based on a dual Lloyd's mirror interferometer. Opt. Commun. 2016, 360, 68-72. [CrossRef]

23. Zhang, J.; Wang, Z.; Di, X.; Zhao, L.; Wang, D. Effects of azimuthal angles on laser interference lithography. Appl. Opt. 2014, 53, 6294-6301. [CrossRef] [PubMed]

24. Stay, J.L.; Gaylord, T.K. Three-beam-interference lithography: Contrast and crystallography. Appl. Opt. 2008, 47, 3221-3230. [CrossRef] [PubMed] 